Instituto Internacional de Investigación y Desarrollo Tecnológico Educativo INDTEC, C.A.

DOI: https://doi.org/10.29394/scientific.issn.2542-2987.2017.0.0.20.376-393

OAI-PMH: http://www.indteca.com/ojs/index.php/Revista Scientific/oai

\title{
Líneas Estratégicas de Comunicación en el Desarrollo de Habilidades Gerenciales y Humanas
}

\author{
Autor: Rider Antonio Moreno Guillen \\ Universidad Pedagógica Experimental Libertador, UPEL \\ riderguillen79@gmail.com \\ Barinas, Venezuela
}

\section{Resumen}

El objetivo del presente estudio es proponer líneas estratégicas de comunicación en el desarrollo de habilidades gerenciales y humanas en el Núcleo Escolar Rural (NER) 187, parroquia Calderas, municipio Bolívar del estado Barinas. La naturaleza del estudio se insertó en el paradigma cuantitativo, el tipo de investigación en la modalidad de Proyecto Factible y el diseño de la investigación se centró en un estudio de campo. La población y la muestra estuvieron conformadas por treinta (30) docentes. Para la recolección de la información se diseñó una encuesta tipo cuestionario con escalamiento Likert con cinco (05) alternativas de respuestas: Siempre, Casi Siempre, Algunas Veces, Casi Nunca y Nunca. El estudio concluye: con poca frecuencia se desarrolla el estilo de comunicación pasiva para el desarrollo de habilidades gerenciales y humanas. Consideran que medianamente el gerente utiliza el estilo de comunicación agresiva para desarrollar las habilidades gerenciales. Además, carece del empleo de la comunicación asertiva para desarrollar las habilidades gerenciales. Así mismo, carecen de las habilidades técnicas, habilidades humanas y las habilidades conceptuales. Estos son relevantes para la propuesta de líneas estratégica en comunicación porque accederá al fortalecimiento de las habilidades gerenciales y humanas tanto del docente como gerente escolar.

Palabras claves: líneas estratégicas; comunicación; habilidades gerenciales. 


\title{
Strategic Lines of Communication in Development of Management and Human Skills
}

\begin{abstract}
The objective of this study is to propose strategic lines of communication in the development of managerial and human skills at the Rural School Nucleus (NER) 187, Calderas parish, Bolívar municipality of Barinas state. The nature of the study was embedded in the quantitative paradigm, the type of research in the Feasible Project modality and the research design focused on a field study. The population and the sample were made up of thirty (30) teachers. For the collection of information, a questionnaire survey was designed with Likert scaling with five (05) response alternatives: Always, Almost Always, Sometimes, Almost Never and Never. The study concludes: infrequently the passive communication style is developed for the development of managerial and human skills. They consider that moderately the manager uses aggressive communication style to develop managerial skills. In addition, it lacks the use of assertive communication to develop managerial skills. Likewise, they lack technical skills, human skills and conceptual skills. These are relevant for the proposal of strategic lines in communication because it will accede to the strengthening of the managerial and human abilities of both the teacher and the school manager.
\end{abstract}

Keywords: strategic lines; communication; managerial skills.

Date Received: 28-11-2016

Date Acceptance: 21-01-2017 


\section{A modo de Introducción}

Por naturaleza el ser humano mantiene una serie de expectativas y deseos, que le son propias de su condición, estas les sirven como mecanismo de búsqueda constante de su bienestar y mantenimiento en procesos de adaptación, de los cuales su individualidad lo orienta hacia sus logros.

El hombre, al integrarse a un medio laboral, busca cumplir con las normas establecidas y brindar sus potencialidades, actitudes y destrezas, a manera de llenar las formalidades en función del perfil característico de un cargo cumpliendo con las necesidades del puesto de trabajo. En ese mismo sentido, éste busca a su vez llenar sus expectativas de superación y satisfacer necesidades individuales dentro de la organización.

En cada uno de estos procesos los gerentes buscan líneas estratégicas para tomar decisiones acertadas con habilidad y competencia, contribuyendo de esta manera a orientar el proceso educativo con eficiencia y eficacia en los niveles y modalidades educacionales. Los directivos de las escuelas primarias, son los primeros llamados a orientar con estrategias para lograr la interacción armónica con el entorno institucional y con el contexto sociocultural; tomar decisiones, trabajando de manera conjunta hacia la consecución, con calidad, de los objetivos institucionales y mejorar el desempeño de todos.

Dentro de los aspectos más importantes de las relaciones entre las personas se encuentra la comunicación, definida como el proceso donde se establecen lazos que implican conductas honestas, respetuosas y positivas cuando se interactúa con otras personas, sin embargo cada ser humano posee temperamento y carácter individual donde se pueden reflejar los tipos de comunicación asertiva (asertiva, pasiva y agresiva); ya que a través de ella se logra intercambiar ideas, experiencias y valores; transmitir sentimientos, actitudes y conocerse mejor. La comunicación permite expresarse y saber más de sí mismo, de los demás y del medio en que se vive. La comunicación sirve para expresar lo que se siente, lo que necesita, lo que se piensa de los demás, 
para entender al mundo que lo rodea, para ser escuchados; también para conocer a las personas con las que se relacionan diariamente.

Es por ello, que la línea estratégica de comunicación para el fortalecimiento de las habilidades gerenciales; van de la mano simultáneamente para producir un ambiente armónico, cooperativo y de negociación entre los miembros involucrados que conviven en una institución educativa, para así lograr el objetivo final, que servirá en función del desempeño óptimo de la organización y la motivación necesaria para cada elemento participante en dicha realidad.

Partiendo de lo antes expuesto, surge la inquietud de realizar un trabajo de investigación que tiene como objetivo proponer líneas estratégicas de comunicación en el desarrollo de habilidades gerenciales y humanas en el Núcleo Escolar Rural (NER) 187, parroquia Calderas, municipio Bolívar del estado Barinas.

La persistencia de esta situación puede ocasionar la deslegitimación de los procesos gerenciales, obstaculizar el cumplimiento de las tareas propuestas para el logro de los objetivos institucionales, así como ser intolerable la convivencia entre los docentes y el directivo, es por ello, que se hace necesario fomentar estrategias gerenciales que facilite la comunicación efectiva entre los docentes y el directivo, apuntando hacia el logro de una organización eficiente en pro de la calidad de los procesos de enseñanza y aprendizaje. Partiendo de esta reflexión surge la siguiente interrogante que orientan la investigación:

1. ¿Cuál es la situación existente en cuanto a la aplicación de líneas estratégicas de comunicación en el desarrollo de habilidades gerenciales y humanas por los docentes y directivos del Núcleo Escolar Rural $187 ?$ 


\subsection{Objetivo General}

Proponer líneas estratégicas de comunicación en el desarrollo de habilidades gerenciales y humanas en el Núcleo Escolar Rural (NER) 187, parroquia Calderas, municipio Bolívar del estado Barinas.

\section{Teorías y Conceptos}

Los antecedentes son conocimientos generados por investigaciones anteriores con respecto a una misma situación, pero los mismos han sido efectuados en diferentes unidades de estudios tiempo. Al respecto, Claret (2014) expresa que, "se refieren a la revisión de trabajos previos sobre el tema en estudio realizados fundamentalmente en instituciones de educación superior reconocidas" (pág. 19). Resumiendo lo tratado, se buscaron indagaciones que guardaban estrecha relación con la temática de estudio de modo que fuera pertinente para la indagación, por lo que fue significativo para la misma porque se recopilaron varios antecedentes que guardaron relación con la indagación. Por lo tanto, en el presente estudio los antecedentes están relacionados con las líneas estratégicas de comunicación en el desarrollo de habilidades gerenciales y humanas.

En este sentido, Uzcategui (2015), en su estudio titulado Comunicación Asertiva como Herramienta Fortalecedora de las Relaciones Interpersonales en el Personal Docente del Liceo Bolivariano "Guillermo Tell Villegas Pulido" Municipio Cruz Paredes, Estado Barinas. Se propuso como objetivo proponer la comunicación asertiva como herramienta fortalecedora de las relaciones interpersonales del personal docente del Liceo Bolivariano "Guillermo Tell Villegas Pulido" del Municipio Cruz Paredes, del Estado Barinas.

Así mismo, la indagación se insertó en el paradigma cuantitativo, para lo cual se ejecutó la modalidad del proyecto factible, con las etapas de diagnóstico, viabilidad y diseño. Se apoyó en el diseño de campo, en vista que se aplicó un instrumento de recolección de datos, organizado por ítems 
respondiendo a las alternativas siempre, casi siempre, algunas veces y nunca, a quince (15) docentes. Las conclusiones permitieron señalar las debilidades de los docentes para desarrollar la habilidades comunicacionales, expresivas e informativas, así como controlar su expresión emocional, por la poca manifestación de los valores que le componen, debilitando los procesos comunicacionales educativos.

El antecedente, antes mencionado, aportó elementos teóricos relacionados con la variable comunicación asertiva y las relaciones interpersonales que son importantes en el desarrollo de la institución. Asimismo, se tomaron aspectos en función al tipo de investigación, el cual estuvo centrado en la modalidad de Proyecto Factible y la técnica e instrumento de recolección de datos utilizados por la investigadora

En este orden y dirección, Soto (2015) en su estudio de Maestría de la Universidad del Zulia, titulado "Las Habilidades Gerenciales y Humanas de los Directores". El objetivo general estuvo enmarcado en describir la gestión y dar una propuesta de evaluación institucional en el contexto de Educación Primaria de la Escuela Bolivariana "Valle de la Pascua", Estado Guárico. Se partió de un diagnóstico inicial el cual arrojo información sobre las condiciones reales de los gerentes educativos dentro de las direcciones de las escuelas. Se determinaron los factores propuestos que determinan la calidad de la gestión del director en las instituciones educativas.

El autor concluyó evidenciaron poco compromiso, y limitado sentido de pertenencia por parte de los directores. El análisis realizado permitió evidenciar los efectos del plan propuesto para los actores de las escuelas. Esta investigación se relaciona con la presente ya que contiene elementos teóricos que permiten sustentar la naturaleza y direccionalidad de las habilidades gerenciales que debe poseer los directores de las escuelas bolivarianas. 


\subsection{Perspectiva Teórica}

\subsection{Líneas Estratégicas de Comunicación}

Las nuevas tendencias y los cambios dinámicos hacen que las organizaciones se vean en la urgente necesidad de orientarse hacia los cambios tecnológicos. Desde la perspectiva más general, la globalización, la apertura económica y la competitividad son fenómenos nuevos a los que se tienen que enfrentar las organizaciones. En la medida que la competitividad sea un elemento fundamental en el éxito de toda organización, los gerentes o líderes harán más esfuerzos para alcanzar altos niveles de productividad y eficiencia.

En este sentido, Páez (2013) reseña que: "la experiencia dice, que con mucha frecuencia las personas que ocupan cargos en los niveles gerenciales son los primeros enemigos del cambio y los responsables de las prácticas establecidas para lograrlo" (pág. 11). El rol de los gerentes como estimuladores del cambio es menos contradictorio de lo que parece. Sólo ellos tienen la responsabilidad del cambio y tienen que asumirla.

Es decir, que aquellas organizaciones que no se adapten a los cambios que se generan en torno a las instituciones, tienen un periodo corto de vida, lo cual trae como consecuencia que caduque y cierre sus instalaciones. Por lo contrario, las organizaciones que se han sostenido a plenitud se caracterizan por llevar a cabo una visión de sus estrategias que sean innovadoras ante las circunstancias que se les presente, basándose en su realidad, así como también incluyendo tecnologías actualizadas, capacitando y motivando con frecuencia su personal.

De esta manera, en el entorno se encuentran presentes tanto las oportunidades como las amenazas. Las gerencias no pueden permanecer ajenas a los procesos políticos, económicos, sociales y tecnológicos que se presenten en el ambiente al cual responden. 
La esencia de toda línea estratégica es propiciar el cambio, dar lugar a un proceso de toma de decisiones para la movilización de los recursos con que cuenta la organización, para pasar de un estado actual a otro superior y deseado. Con todos los elementos que enriquecen el proceso de dirección, en lo conceptual y metodológico de la planeación y, en particular, los que caracterizan a la estrategia, sus aspectos medulares, sus formas de elaboración, su incidencia y valor en el proceso de dirección.

Por su parte, Ortiz (2013) expone que la línea estratégica toma en cuenta cuatro fases como son: "fijar normas de gestión en la organización; efectuar un registro de gestión individual; establecer una confrontación de la gestión con las normas establecidas y tomar decisiones sobre las acciones para mejorar el proceso" (pág. 41).

En consecuencia, las líneas estratégicas tienen como finalidad la transformación institucional y del entorno, involucrar a todos para ayudar a enfrentar la democratización del proceso, la descentralización y la autonomía, la apertura de la organización, la concepción prospectiva del proceso y los procesos de cambio en el orden económico y social.

\subsection{Comunicación}

La comunicación es un proceso de creación, intercambio, procesamiento y almacenamiento de mensajes dentro de un sistema de objetivos determinados. Valdez (2013), plantea, que la comunicación organizacional no es un proceso que solo se lleve a cabo en el interior de la organización, sino también fuera de ella, pues el buen funcionamiento de sus redes internas debe ser paralelos con las que se tienen con los miembros externos de la misma. El referido autor plantea los siguientes tipos: "comunicación formal, comunicación interna, comunicación descendente, y comunicación ascendente" (pág. 54). 
Es decir, que aquellas organizaciones que no se adapten a los cambios que se generan en torno a las instituciones, tienen un periodo corto de vida, lo cual trae como consecuencia que caduque y cierre sus instalaciones. Por lo contrario, las organizaciones que se han sostenido a plenitud se caracterizan por llevar a cabo una visión de sus estrategias que sean innovadoras ante las circunstancias que se les presente, basándose en su realidad, así como también incluyendo tecnologías actualizadas, capacitando y motivando con frecuencia su personal.

\subsection{Habilidades Personales Gerenciales}

Las habilidades gerenciales del director están asociadas, en primer lugar, a las habilidades personales; las cuales se traducen en las actitudes puestas de manifiesto durante su gestión. Esto lo apoya en una serie de características o cualidades personales que posee el gerente para la conducción de la escuela que dirige. Al respecto, Graffe (2011) expone que entre las habilidades personales del director están las siguientes competencias asociadas: "conocimiento de sí mismo, autorrealización y autocontrol" (pág. 74).

Por lo anteriormente expuesto, es de suma importancia tener en cuenta que se necesitan ciertas habilidades generales para tener un desempeño directivo eficaz, sin importar que nivel en jerarquía se ocupe en la organización. Por otro lado, es importante precisar que la mezcla de habilidades que debe tener un directivo varía de acuerdo al contexto en el cual se desarrolle una institución

\section{Marco Metodológico}

El marco metodológico según Balestrini (2008), "está referido al conjunto de procedimientos lógicos, tecno-operacionales implícitos en todo proceso de investigación, con el objetivo de ponerlo en manifiesto y 
sistematizarlos, a propósito de permitir, descubrir y analizar los supuestos del estudio y reconstruir los datos" (pág. 125). De acuerdo a lo planteado, en este capítulo se describe, atendiendo los objetivos del estudio las fases que lo orientan, la naturaleza de la investigación que permita la operatividad del estudio y comprobar su factibilidad o posibilidad para su realización.

\subsection{Naturaleza de la Investigación}

De acuerdo a los objetivos formulados la investigación se enmarcó en el paradigma cuantitativo. Hernández, Fernández y Baptista (2010), plantean que dicho paradigma:

Utiliza la recolección y el análisis de datos para contestar preguntas de investigación y probar hipótesis establecidas previamente, y confía en la medición numérica, el conteo y frecuentemente en el uso de las estadísticas en una población para establecer con exactitud patrones de comportamiento en una población (pág. 5).

Es decir, se requiere que entre los elementos del problema de investigación exista una relación cuya naturaleza sea lineal; que haya claridad entre los elementos del problema de investigación que conforman el problema, que sea posible definirlo, limitarlos y saber exactamente donde se inicia el problema, en cual dirección va y que tipo de incidencia existe entre sus elementos. De esta manera los postulados y principios se apoyan en la corriente positivista, en virtud de considerar que el conocimiento debe ser organizado y clasificado.

\subsection{Tipo de Investigación}

La investigación ayuda a mejorar el estudio porque permite establecer contacto con la realidad a fin de que se conozca mejor. Constituye un estímulo para la actividad intelectual creadora. Ayuda a desarrollar una curiosidad 
creciente acerca de la solución de problemas. Contribuye al progreso de la lectura crítica

En este sentido, el tipo de investigación, para el presente estudio, se enmarcó en la modalidad de Proyecto Factible. Al respecto, Arias (2012) lo define como "una propuesta de acción para resolver un problema práctico o satisfacer una necesidad. Es indispensable que dicha propuesta se acompañe de una investigación que demuestre su factibilidad o posibilidad de realización" (pág. 112). Atendiendo este criterio, el Proyecto Factible constituye una propuesta que se dirige a la identificación de los elementos esenciales de la problemática y su posterior articulación de factores de mejoramiento, con base en la confrontación de los hechos en la realidad que les circunda.

Por lo tanto, a través del presente estudio se pretende colocar a disposición de la población en estudio, una propuesta sobre líneas estratégicas de comunicación en el desarrollo de habilidades gerenciales y humanas en el Núcleo Escolar Rural 187, parroquia Calderas, municipio Bolívar del estado Barinas; brindando una solución viable a la problemática planteada.

\subsection{Diseño de la Investigación}

El diseño de investigación se centró en un estudio de Campo. Arias (2012), la define: "son aquellas que consisten en la recolección de datos directamente de los sujetos investigados o de la realidad donde ocurre el hecho (datos primarios)" (pág. 31). La investigación de campo se caracteriza por recoger datos a través de fuentes primarias, además analiza procedimientos estadísticos. Por el planteamiento del objetivo en el cual se centrará la investigación, se trata de una investigación de campo-descriptiva, debido a que la información se obtiene de la realidad y se basa en la observación directa. Por lo tanto, se recolectarán datos directamente de la realidad. 
Igualmente, Incluye descripción, registro, análisis e interpretación de la naturaleza actual, composición o procesos de los fenómenos. Es decir, hace énfasis sobre conclusiones dominantes o sobre cómo una persona, grupo o cosa se conduce o funciona en el presente. Además, está dirigida al conocimiento del presente, a encontrar respuesta a los problemas teóricos y prácticos que tejen la trama educativa.

La población o universo es el grupo al cual el investigador trata de explicar generalizaciones obtenidas e intenta formular afirmaciones que sean valederas para ese conglomerado o grupo total. Al respecto, Martin (2012) define la población como: "la representación de todos los elementos de forma parcial que intervienen directa e indirectamente en la investigación". (pág. 45). Es decir, representa el conjunto de elementos o personas sujetas a estudio. El presente estudio se tomó una población de treinta (30) docentes y directivos que laboran en el Núcleo Escolar Rural 187, parroquia Calderas, municipio Bolívar del estado Barinas, lo cual es una población importante y protagonista para el objeto de estudio.

La muestra, según Hernández, Fernández y Baptista (2010) representa "un subgrupo de la población... es un subconjunto de elementos que pertenecen a ese conjunto definido en sus características al que llamamos población" (pág. 240). De acuerdo a las características que presenta la población: pequeña y finita, no se utilizaran criterios muéstrales, por lo tanto, se consideró la totalidad de ésta, quedando conformada treinta (30) sujetos de estudio.

Por lo tanto, la muestra se considera censal, razón por la cual se seleccionó el $100 \%$ de la población al considerarla un número manejable de sujetos. En este sentido, Ramírez (2012) establece la muestra censal es aquella donde todas las unidades de investigación son consideradas como muestra. De allí, que la población a estudiar se precise como censal por ser simultáneamente universo, población y muestra. 
Las técnicas de recolección de datos constituyen las diferentes formas en que se recogerán la información, necesario para llevar a cabo la investigación. La técnica utilizada fue la encuesta que, según Arias (2012), es "una técnica que pretende obtener información que suministra un grupo o muestra de sujeto acerca de si mismo, o en relación a un tema en particular" (pág. 72), esta técnica facilitó la obtención de datos exactos y confiables de los estudiantes a través del contacto con la realidad, contribuyendo en la realización del diagnóstico que describe la situación objeto de estudio.

En función de la encuesta, se empleó como instrumento el cuestionario, que Fernández (2012) dice que en éste "las preguntas escritas son respondidas por el informante sin que se requiera necesariamente la presencia del investigador" (pág. 90), por lo tanto, se entregará cada una de las encuestas y se recogió en el momento que se consideró oportuno, por parte de la investigadora y los sujetos de la muestra. El instrumento que se utilizó fue el cuestionario con escalamiento tipo Lickert.

Finalmente, los datos recolectados se codificaron, clasificaron y tabularon, para ser graficados, analizados y explicados e inferir a partir de ellos la necesidad de elaboración de la propuesta de líneas estratégicas de comunicación, desde la perspectiva de Stephen Covey en el desarrollo de habilidades gerenciales y humanas en el Núcleo de Educación Rural 187, parroquia Calderas, municipio Bolívar del estado Barinas y de los planteamientos que se formularon a partir de las variables.

\section{Conclusiones y Recomendaciones}

\subsection{Conclusiones}

La culminación de la investigación lleva a establecer como conclusiones, respecto al comportamiento reflejado por la muestra y que es generalizado a toda la población en función al objetivo de diagnosticar la situación existente en cuanto a la aplicación de líneas estratégicas de 
comunicación en el desarrollo de habilidades gerenciales y humanas por los docentes y directivos del Núcleo Escolar Rural 187, el mismo determinó lo siguiente:

El equipo directivo emplee el proceso de comunicación en los docentes para dar una efectiva información. Además, necesitan utilizar el emisor y receptor para intercambiar mensajes en el ámbito laboral; así como también tienen ausencia de emplear la conexión cara a cara como medio de comunicación entre los compañeros de trabajo. Igualmente, ameritan una transmisión efectiva de comunicación con el equipo directivo. No se utilizan las ideas e informaciones de manera efectiva con los docentes.

Los docentes opinaron con poca frecuencia se desarrolla el estilo de comunicación pasiva para el desarrollo de habilidades gerenciales y humanas. Consideran que medianamente el gerente utiliza el estilo de comunicación agresiva para desarrollar las habilidades gerenciales. Además, carece del empleo de la comunicación asertiva para desarrollar las habilidades gerenciales.

Los encuestados consideran que carecen de las habilidades técnicas, habilidades humanas y las habilidades conceptuales. Estos son relevantes para la propuesta de líneas estratégica en comunicación porque accederá al fortalecimiento de las habilidades gerenciales y humanas tanto del docente como gerente escolar.

Los gerentes carecen del conocimiento sí mismo de acuerdo a las necesidades y motivaciones del personal; así como también la autorrealización en la adquisición de los propios ideales y existe ausencia en el control relacionado con el manejo de conflictos. Estos datos son relevantes, para el presente estudio, razón por la cual se proporcionarán líneas estratégicas que contribuirán con el desarrollo de habilidades gerenciales.

El objetivo de determinar la factibilidad, desde lo social, técnica, económica y educativa de la propuesta de líneas estratégicas de comunicación 
en el desarrollo de habilidades gerenciales y humanas, la propuesta es viable, desde el punto de vista económico porque la evaluación de los costos no requiere de grandes ingresos económicos. La factibilidad técnica se cuenta con los recursos materiales y humanos para la implementación de la propuesta

y la factibilidad social busca la formación integral del individuo por medio del reforzamiento de la comunicación y habilidades para la vida.

\subsection{Recomendaciones}

Promover charlas educativas por el equipo directivo para desarrollo los estilos comunicacionales, tales como el pasivo, agresivo y asertivo como estrategia de fortalecimiento en las habilidades gerenciales y humanas.

Realizar diálogos interactivos, entre docentes y directivos, con el propósito que se abordado los tipos de comunicación y su importancia en el desarrollo de las habilidades gerenciales, humanas y otras necesidades de los actores y autores educativos.

Propiciar colectivos de formación relacionado con la comunicación efectiva, desarrollo de habilidades gerenciales, habilidades técnicas, habilidades humanas y habilidades conceptuales.

Planificar, entre docentes y directivos las líneas estratégicas de comunicación, en cual se fijen vías, herramientas, materiales y equipos que fortalezca la comunicación interactiva entre todos los involucrados en el hecho educativo, como clave fundamental para lograr el desarrollo de habilidades gerenciales.

Formular y ejecutar estrategias para consolidar el trabajo en equipo con todos los trabajadores, con la finalidad de unir esfuerzos para resolver los problemas de una manera mancomunada.

Se recomienda al equipo directivo aplicar las siguientes habilidades gerenciales: emplear el trabajo cooperativo para planificar, ejecutar, evaluar y darle seguimiento a los planes, programas y proyectos, desarrollando 
credibilidad y gobernabilidad. Encontrar un punto de convergencia que permita hacer efectiva la creatividad y el talento de los trabajadores.

\section{Referencias}

Arias, F. (2012). El proyecto de investigación. (6ta ed.). Caracas: Episteme. Balestrini, M. (2008). Como se elabora el Proyecto de Investigación (Para los estudios descriptivos, diagnósticos, evaluativos experimentales y los proyectos factibles). (7ma Ed.). Caracas: BL. Consultores Asociados Servicio Editorial.

Claret, R. (2014). Metodología de la investigación. Colombia: Norma.

Fernández, R. (2012). Tipos de Instrumentos de Medición o Recolección de Datos. Buenos Aires.

Graffe, G. (2011). Gestión Educativa para la Transformación. Caracas: Universidad Central de Venezuela.

Hernández, R. Fernández, C. y Baptista, P. (2010). Metodología de la investigación. México: McGraw-Hill.

Martin C. (2012). Metodología de la Investigación. Caracas: Editorial Romor. Páez, L. (2013). Gerencia. Caracas: Planeta.

Ramírez, F. (2012). Cómo Hacer un Proyecto de Investigación. Editorial Panapo. Caracas.

Soto, L. (2015). Las Habilidades Gerenciales y Humanas de los Directores". El objetivo general estuvo enmarcado en describir la gestión y dar una propuesta de evaluación institucional en el contexto de Educación Primaria de la Escuela Bolivariana "Valle de la Pascua". Estado Guárico. Trabajo de Grado de Maestría, Universidad del Zulia, Maracaibo.

Uzcategui, L. (2015). Comunicación asertiva como herramienta fortalecedora de las relaciones interpersonales en el personal docente del Liceo Bolivariano "Guillermo Tell Villegas Pulido" 
Municipio Cruz Paredes. Estado Barinas. Trabajo de Grado de Maestría, Publicado, Universidad Fermín Toro. Cabudare, Barquisimeto.

Universidad Pedagógica Experimental Libertador (2006). Manual de Trabajos de Grado, Especialización, Maestría y Tesis Doctorales. (4ta ed) FEDUPEL. Caracas.

Valdez, N. (2013). Tipos de comunicación organizacional. Buenos Aires: Editores. 


\section{Rider Antonio Moreno Guillen \\ e-mail: riderguillen79@gmail.com}

Nacido el 07 de septiembre de 1985, natural de Barinas,

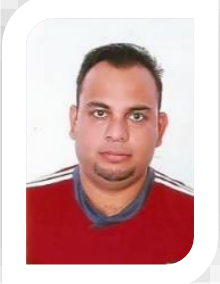
Venezuela, 31 años de edad, soltero, dirección: Barinitas, municipio Bolívar, estado Barinas. Estudios: Primaria: Escuela Básica José Vicente Unda. Secundaria: 7 mo a 5to año Colegio Provincial Barinitas. Pregrado: Profesor Especialidad Ingles en la Universidad Pedagógica Experimental Libertador, Barinas. Postgrado: Cursante Maestría: Gerencia Educacional, UPEL, Barinas. Desempeño: Docente de Ingles: Escuela Básica Bolivariana "Los Naranjos" Calderas, Barinitas, 2008-2013. Coordinador de Defensoría Educativa "Semillas del Futuro", En La Parroquia Calderas, 20132016. Docente Especialidad Ingles Liceo Bolivariano Juana Ramírez La Avanzadora Barinitas, 2016.

El contenido de este manuscrito se difunde bajo una Licencia de Creative Commons ReconocimientoNoComercial-Compartirlgual 4.0 Internacional 